\title{
Degradation effect on the magnetoresistance in organic light emitting diodes
}

Tobias D. Schmidt*, Andreas Buchschuster, Matthias Holm, Stefan Nowy, Josef A. Weber, Wolfgang Brütting*

University of Augsburg, Institute of Physics, Universitätsstr. 1, 86135 Augsburg, Germany

\begin{abstract}
The magnetoresistance in organic light emitting diodes (OLEDs) can be enhanced by electrical stressing of the device. In this study, magnetotransport measurements were performed on pristine and aged organic hetero-layer light emitting diodes based on small molecules with Alq 3 "(tris(8-hydroxyquinoline)aluminum) as emitter. Under pristine conditions the maximum organic magnetoresistance (OMR) was of the order of $1 \%$ at an applied magnetic field of $100 \mathrm{mT}$ and a voltage of $3.5 \mathrm{~V}$. After electrical stressing at a constant current density we observed an increase of the maximum OMR together with a shift towards higher voltages. The maximum OMR reached almost $6 \%$ at an applied magnetic field of $100 \mathrm{mT}$ and a voltage of about $5 \mathrm{~V}$. To verify the correlation between electrical aging and the magnitude of the OMR effect, we investigated OLEDs with different hole injection layers exhibiting significantly different lifetimes.
\end{abstract}

Keywords: Organic magnetoresistance, Organic light emitting diode,

\footnotetext{
*Corresponding author.

Email addresses: Tobias.Schmidt@physik.uni-augsburg.de (Tobias D. Schmidt), Wolfgang.Bruetting@physik.uni-augsburg.de (Wolfgang Brütting)
} 
Magnetic field effect, Degradation

\section{Introduction}

The interest of research in organic light emitting diodes (OLEDs) has grown continuously since the first efficient OLED was presented by Tang and VanSlyke in 1987 [1]. In recent years it has been reported, that a weak magnetic field has a strong influence on the electrical behavior and the electroluminescence of OLEDs $[2,3,4]$. It has been shown, that the resistance of the device can be changed considerably by fields of only a few ten $\mathrm{mT}$ [5]. This so called organic magnetoresistance (OMR) has been suggested to be applicable in magnetic field sensors or touchscreen displays, which only interact with a magnetic pen. These devices could benefit from the low-cost and easy processing of OLEDs due to the possibility of simple device layout and evaporation or even printing of the organic layers. Also the option to use flexible substrates is very interesting for future appliances. The requirements for these devices are a strong modification of the current at a constant voltage due to the applied magnetic field at room temperature and long-term stability of this effect. In this context it is notable that both requirements are not yet fulfilled and a better understanding of these effects is necessary to optimize them. For example, there are reports of current changes between $1 \%$ [6] and $20 \%$ [7] for samples based on the small molecule $\mathrm{Alq}_{3}$ (tris(8hydroxyquinoline)aluminum) under nominally identical conditions. Further investigations showed that the OMR is influenced by degradation of the device due to electrical stressing $[6,8]$. In polymer based OLEDs this effect was enhanced from $1 \%$ to more than $15 \%$ due to electrical stressing at a 
constant current for one hour.

The real mechanism behind the enhanced OMR resulting from degradation is still not well understood, but the elucidation of correlations between both phenomena could improve the comprehension of the magnetic field effects in organic light emitting diodes.

\section{Experimental}

The devices used in this study have an active area of $4 \mathrm{~mm}^{2}$ and have a typical structure that is shown in Fig. 1. First of all, the hole injection layer (HIL) has been spincast on the ITO (indium tin oxide) coated glass substrates. In our experiment two different HILs are used. Both HILs, poly(3,4-ethylenedioxythiophene):poly(styrenesulfonate) (PEDOT:PSS, Clevios P AI4083) and HIL1.3 (Clevios HIL1.3, structure unknown) are commercial materials from H.C. Starck Clevios and exhibit significantly different device lifetimes in OLEDs [9]. Second, the hole transport material N,N'di(naphtalene-1-yl)-N,N'-diphenyl-benzidine (NPB) and the electron transporter and emitter $\mathrm{Alq}_{3}$, both purchased from Sensient Imaging Technologies

\begin{tabular}{|lr|}
\hline \multicolumn{2}{|c|}{ Encapsulation } \\
\hline $\mathrm{Al}$ & $200 \mathrm{~nm}$ \\
\hline $\mathrm{LiF}$ & $0.3 \mathrm{~nm}$ \\
\hline $\mathrm{Alq}_{3}$ & $60 \mathrm{~nm}$ \\
\hline $\mathrm{NPB}$ & $50 \mathrm{~nm}$ \\
\hline $\mathrm{HIL}$ & $50 \mathrm{~nm}$ \\
\hline ITO & $120 \mathrm{~nm}$ \\
\hline \multicolumn{3}{|c|}{ Glass } \\
\hline
\end{tabular}

Figure 1: Schematic layer stack of the investigated devices. 
GmbH as sublimed grade, together with a LiF:Al cathode have been evaporated in a vacuum chamber with a base pressure of $<5 \cdot 10^{-7}$ mbar. Finally, the devices have been encapsulated in a nitrogen glovebox in order to protect them from ambient air. The complete device fabrication has been described elsewhere [9].

All measurements have been carried out with computer controlled setups in a specially designed box. During the OMR measurements, the magnetic field (Bruker B-E15 V, electro magnet) was oriented parallel to the sample surface. The electrical characterization (I-V-characteristics, degradation, OMR) has been performed with a Keithley 2400 sourcemeter and the electroluminescence was detected by a photodiode (Gigahertz-Optik) coupled with a Keithley 6514 electrometer. For OMR measurements a procedure was performed to minimize the mistake due to the zero-field current drift with time. In this procedure the zero-field current is measured before and after the current measurement with the applied magnetic field at a constant voltage [10]. After calculating the average value of the zero-field current, the normalized current difference with and without the magnetic field $(\Delta I / I=[I(B)-I(0)] / I(0))$ is analyzed. This is commonly called OMR, although strictly speaking the change of the resistance $\Delta R / R$ is the inverse of $\Delta I / I$. Furthermore the effect of the magnetic field on the electroluminescence has been measured. We call this change OML (organic magneto-luminescence) and it is calculated analogous to the OMR from $[E L(B)-E L(0)] / E L(0)$. 


\section{Results and Discussion}

Both types of samples, those with PEDOT:PSS and those with HIL1.3 as hole injection layer, have similar electro-optical characteristics under pristine conditions (see Fig. 2). Although HIL1.3 and PEDOT:PSS devices have quite the same I-V-curves as well as luminance intensities under a given voltage, the samples show very different degradation behavior at identical stressing conditions $\left(75 \mathrm{~mA} / \mathrm{cm}^{2}\right)$. As it can be seen in Fig. 3 devices with PEDOT:PSS degrade much faster than devices with HIL1.3 as hole injection

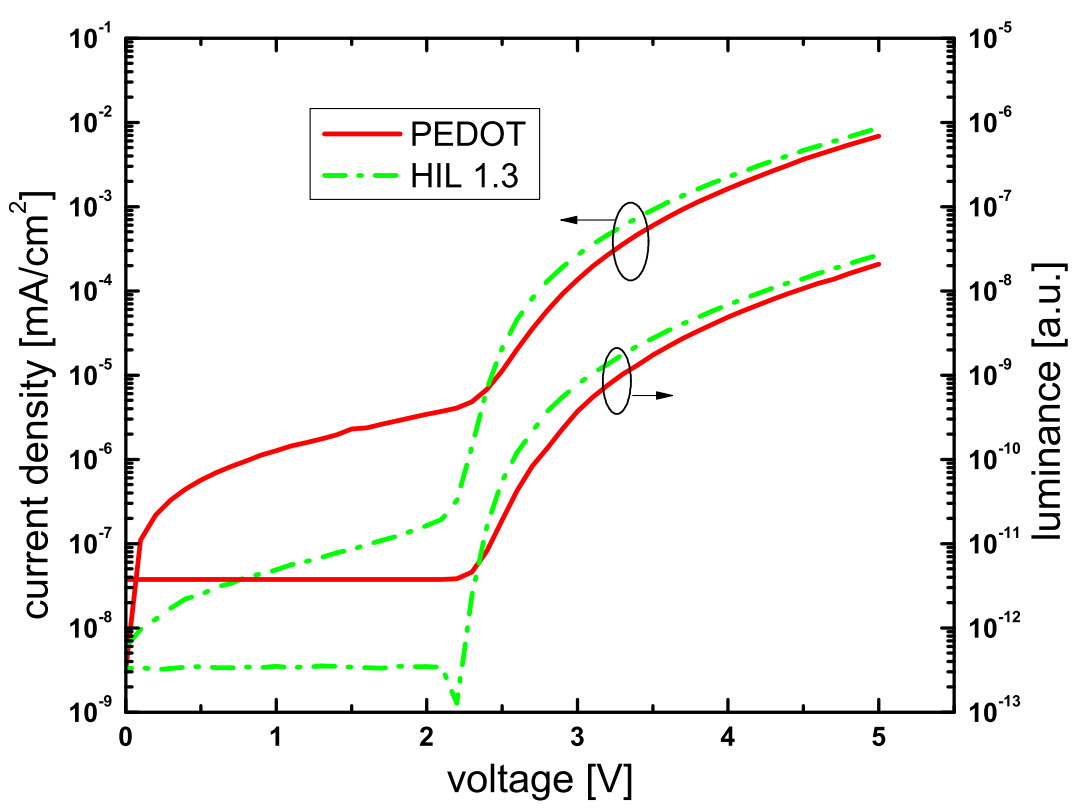

Figure 2: Current-voltage and luminance-voltage characteristics for pristine ITO/HIL/Alq $/ \mathrm{LiF} / \mathrm{Al}$ devices with PEDOT:PSS (red) and HIL1.3 (green) as hole injection layers. 
layer. The luminance of the PEDOT:PSS device drops to $30 \%$ (LT 30) of its pristine value already after 36 hours aging time, while it takes 500 hours for the HIL1.3 device to drop to this luminance level.

It should be noted that the devices only differ in their hole injection layer. Device structure and all other organic layers are the same. Because a mono-exponential fit could not satisfactorily reproduce the observed decay of the luminance with stressing time, a bi-exponential fit was carried out for both devices. We therefore assume that there are two different degradation effects. As can be seen in Table 1, the fitting parameters of both devices are comparable with the exception of one time constant. This time constant is for PEDOT:PSS devices about 60 times smaller than for HIL1.3 devices, which means that one of the two degradation effects is more pronounced in PEDOT:PSS devices. We note that a detailed study of the degradation of the two types of devices by impedance spectroscopy has previously been published by our group [9]. These and other investigations have revealed that the degradation of the luminescence is mainly caused by generation of nonradiative recombination centers $[12]$ at the $\mathrm{NPB} / \mathrm{Alq}_{3}$ interface forming a positive interfacial charge. It is believed to originate from a material specific instability at or close to this interface, e.g. the instability of cationic $\mathrm{Alq}_{3}$ species [22]. A second possible degradation process is the instability of the HIL/NPB interface including trap formation in the NPB layer or at the HIL/NPB interface, which is more pronounced in devices with hydrophilic hole injection layers, i.e. PEDOT:PSS [9]. This could be an effect that also influences the OMR behavior with aging time, as will be discussed below.

Under pristine conditions PEDOT:PSS and HIL1.3 devices showed an 


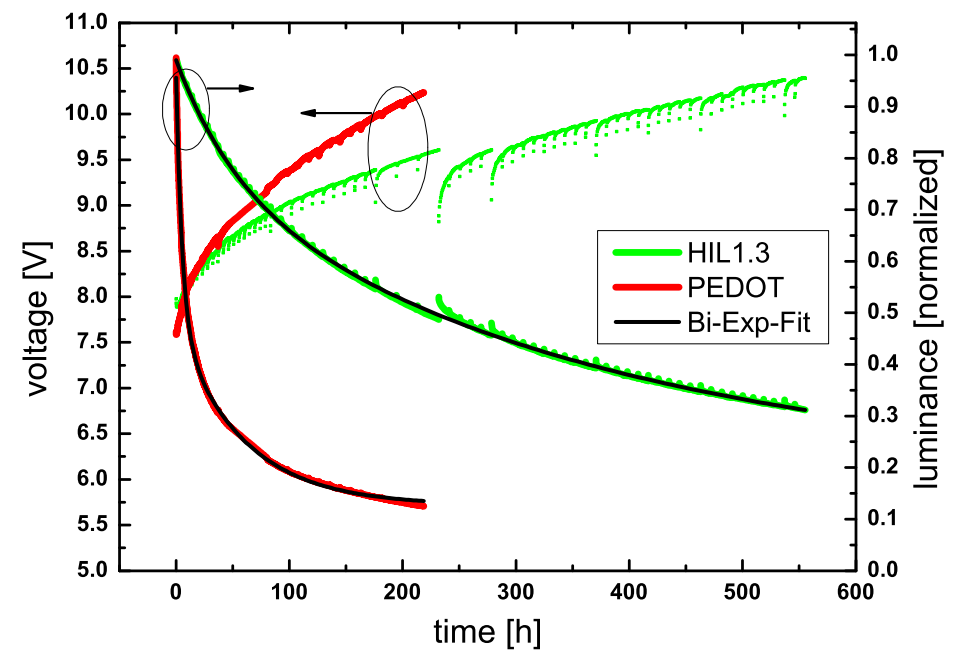

Figure 3: Decrease of the electroluminescence intensity and increase of the applied voltage for both types of samples with PEDOT:PSS (red) and HIL1.3 (green) at constant current driving $\left(j=75 \mathrm{~mA} / \mathrm{cm}^{2}\right)$. Especially for the sample with HIL1.3 partial recovery is seen due to interruptions of the aging process for a couple of hours. The black lines are biexponential fits of the electroluminescence decrease with aging time. See Table 1 for more information about the fit parameters.

Table 1: Fitting parameters of the biexponential decrease of the electroluminescence shown in figure 3.

\begin{tabular}{|c|c|c|}
\hline Fit parameters & PEDOT:PSS & HIL1.3 \\
\hline \hline$y_{0}$ & 0.13 & 0.18 \\
\hline$A_{1}$ & 0.48 & 0.59 \\
\hline$t_{1}[\mathrm{~h}]$ & 5.45 & 371.29 \\
\hline$A_{2}$ & 0.35 & 0.29 \\
\hline$t_{2}[\mathrm{~h}]$ & 58.25 & 52.45 \\
\hline
\end{tabular}



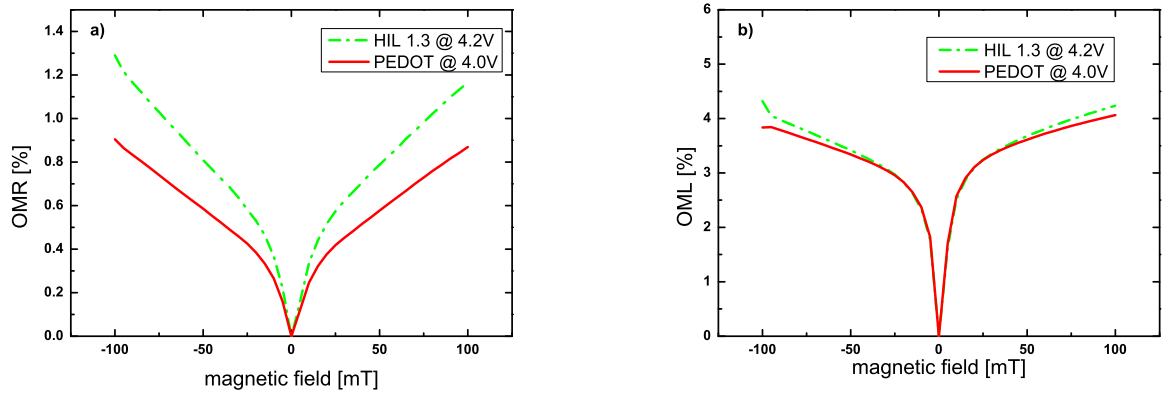

Figure 4: Influence of a magnetic field on pristine devices with PEDOT:PSS (red) and HIL1.3 (green) as HIL. Magnetic field effect at a fixed voltage a) on the resistance and b) on the electroluminescence.

OMR of about $0.9 \%$ and $1.2 \%$ at a magnetic field of $100 \mathrm{mT}$ and at voltages of $4.0 \mathrm{~V}$ and $4.2 \mathrm{~V}$, respectively, and the OML is about $4 \%$ for both devices at the same voltages as the OMR (Fig. 4). Interestingly the OMR for both samples does not saturate even at a magnetic field (B) of $100 \mathrm{mT}$, whereas the OML almost reaches a plateau. Most OMR vs. B measurements published in literature show a different behavior. Typically the OMR reaches a plateau with increasing magnetic field, while the OMR vs. B measurements presented here exhibit a linear behavior even at $100 \mathrm{mT}$. This is similar to measurements of Desai et. al [13] and Shakya et. al [21], who explained that behavior with material parameters like the purity of the $\mathrm{Alq}_{3}$ layer. However, after electrical stressing of the devices, higher values of the OMR are reached and the shape of the OMR vs. B measurement changes to the typical behaviour (not shown here). This could be an indication of trap formation in the HTL.

Fig. 5 shows the behavior of the OMR for the PEDOT:PSS and the 

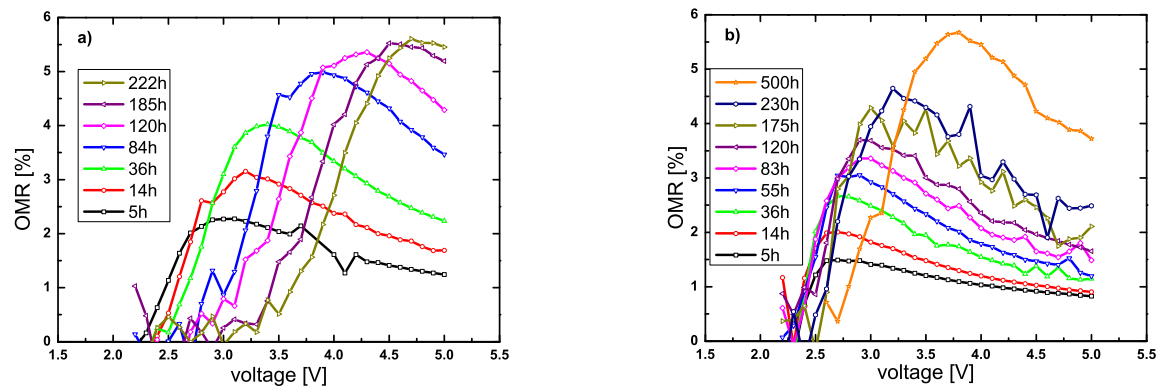

Figure 5: OMR vs. voltage at a magnetic field of $100 \mathrm{mT}$ a) for devices with PEDOT:PSS and b) for devices with HIL1.3 at different aging times.

HIL1.3 devices at a constant magnetic field of $100 \mathrm{mT}$ for different aging times and for voltages between $2.0 \mathrm{~V}$ and $5.0 \mathrm{~V}$. When the magnetic field is kept constant, the OMR has a broad maximum at a certain voltage. With stressing time the height of this maximum increases and its position shifts to larger voltages. Both samples show this behavior, but the increase of the OMR associated with the shift of the maximum is much faster in PEDOT:PSS samples. For example after 120 hours stressing time the PEDOT:PSS devices have a maximum OMR of $5.4 \%$ at a voltage of $4.3 \mathrm{~V}$, while the HIL1.3 devices only show a maximum OMR of $2.9 \%$ at a voltage of $3.7 \mathrm{~V}$. The observed voltage shift, as shown in Fig. 6, is for both samples almost linear with time. It is also visible that the maximum of the OMR increases very fast for the PEDOT:PSS devices and then remains constant at a value of $5.6 \%$ for stressing times longer than 150 hours, while the increase of the OMR for the HIL1.3 devices is much slower and achieves $5.6 \%$ not until an aging time of 500 hours.

The voltage dependent OML measurements at different aging times for 

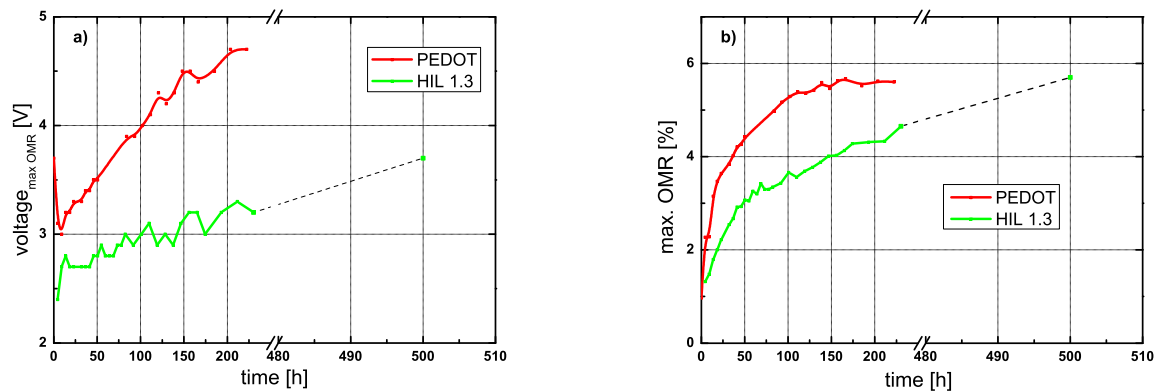

Figure 6: a) Shift of the voltage of the maximum OMR with aging time for PEDOT:PSS (red) and HIL1.3 (green) devices. b) Increase of the maximum OMR with aging time for PEDOT:PSS (red) and HIL1.3 (green) devices.

both types of samples are shown in Fig. 7. Under pristine conditions both devices do not show a maximum in the OML curves, but this behavior changes with stressing time. Already after a very short aging time of $14 \mathrm{~h}$ a maximum in the OML appears for the PEDOT:PSS devices. The magnitude of the maximum remains quite constant at $8 \%$ but it shifts to higher voltages. For HIL1.3 devices the magnitude of the OML is higher (12\% at LT 30) than for PEDOT:PSS devices (8\% at LT 30) although the OMR has comparable values at LT 30 for both samples. Both the maximum of the OML and the maximum of the OMR are located at similar voltages for a certain stressing time. This is reasonable because the current at a fixed voltage is increased by the magnetic field. However it is believed, that the occurrence of an OMR is not the only reason for the OML effect, otherwise the maximum in the OML should exhibit the same magnitude and exactly the same position as the OMR in the absence of exciton polaron quenching. One problem is the measured OML behaviour for low current densities at voltages about $2.5 \mathrm{~V}$. 

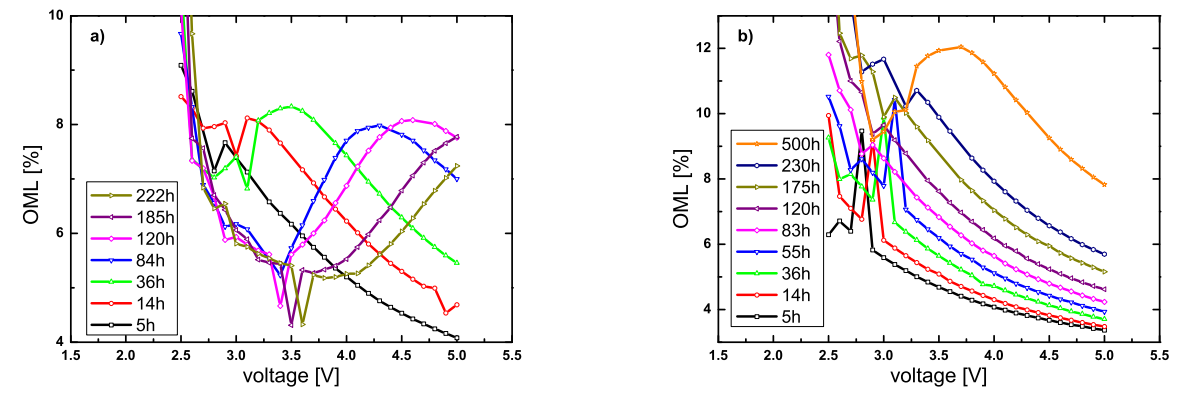

Figure 7: OML vs. voltage at a fixed magnetic field of $100 \mathrm{mT}$ a) for devices with PEDOT:PSS and b) for devices with HIL1.3 at different aging times.

In this range an OMR is well detectable but the measured electroluminescence signal is very low and noisy, so that it is impossible to give an evidence if there is a maximum in the OML or not. Problably there are maxima in the OML for both types of samples even at pristine conditions and also for the HIL1.3 devices below a stressing time of $500 \mathrm{~h}$. However, especially for long degradation times, the voltages at which the maxima of the OML and the OMR appear differ by $0.4 \mathrm{~V}$ for PEDOT:PSS devices. Thus, a direct correlation between the appearance of the maximum of the OML and the decrease of the electroluminescence and especially the appearance of the OMR was not possible.

Other measurements have shown that aging due to electrical stressing is accompanied by a decrease in the hole mobility of the device and by an increase of the interfacial charge between the NPB and the $\mathrm{Alq}_{3}$ interface[9]. The decrease of the hole mobility is due to the formation of traps [11] or increased disorder [23] in the HTL and has been detected via impedance spectroscopy [9]. Based on these results, a possible origin of the OMR in 
OLEDs could be the influence of the magnetic field on the interaction between traps in the NPB layer $[19,20]$ and the dominant charge carrier species in the device, i.e. the holes. The increase of the interfacial charge is mainly due to the formation of cationic radicals. These radicals carry a magnetic moment due to unpaired spins and thus can interact with the external magnetic field leading to an additional contribution to the OML.

Finally, we discuss the investigated effects in the context of existing models for the OMR reported in literature. In the bipolaron model $[14,15]$ a positive effect of the magnetic field on the current through the device is explained by the formation of bipolarons and thus an increase of the resistance if a magnetic field is applied to the device. Due to the continuous formation of traps or increased disorder induced by electrical stressing, more bipolarons could be formed in the device [16] and so the OMR would be increased by electrical stressing, what is in agreement with the measured behavior.

Another reported model is the triplet site blocking mechanism [17]. In this model free polarons interact with triplet excitons in the emission layer and get scattered. One of the supposed degradation mechanisms in this paper is the formation of nonradiative recombination centers which decrease the number of triplet excitons so that less charge carriers get scattered. This is also in agreement with our observations but should only appear close to the emission zone.

The third common description is the so called electron-hole-pair model [18]. In this model a positive effect on the resistance by the applied magnetic field is due to a decrease of the intersystem crossing rate and therefore an increase of the triplet exciton concentration. Triplet excitons mainly disso- 
ciate and thereby generate free charge carriers that can enhance the current flow through the device. With degradation of the device this dissociation is accelerated due to nonradiative recombination centers.

In summary, all common models describing the OMR effect can partially explain our findings. Nevertheless, a conclusive explanation of the observed changes of OMR and OML with device aging and the correlation to other measurements requires further investigations.

\section{Conclusion}

To summarize, magnetotransport measurements on organic light emitting diodes have been performed. The effect of the OMR was enhanced by electrical stressing of the samples at least by a factor of five. Although the degradation behavior of both types of samples is different, there is a clear indication that the decrease of the electroluminescence efficiency and the increase of the maximum OMR are correlated. Though there is no unambiguous evidence yet, that the OMR is caused by the formation of traps in the hole transporting layer, the comparison to results obtained by impedance spectroscopy suggest this scenario. Moreover, since there is no one-to-one correspondence between OMR and OML in these samples, we suppose an additional mechanism to be responsible for changes of the electroluminescence in a magnetic field. One possible effect could be the formation of charged nonradiative recombination centers as detected again by impedance spectroscopy. All in all, we anticipate that a microscopic elucidation of the degradation of OLEDs could also improve the understanding of the magnetic field effects in these devices, and vice versa. 


\section{Acknowledgments}

The authors would like to thank H.C. Starck Clevios, especially Andreas Elschner and Wilfried Lövenich, for providing samples. We also thank the German Federal Ministry of Education and Research (BMBF) as well as the German Research Foundation (DFG) for funding part of this work under contract nos. FKZ 13N10474 (TOPAS) and Br 1728/6, respectively.

\section{References}

[1] C. W. Tang and S. A. VanSlyke, Appl. Phys. Lett. 51(12) (1987) 913915.

[2] J. Kalinowski, M. Cocchi, D. Virgili, V. Fattori, P. di Marco, Phys. Rev. B 70 (2004) 205303.

[3] J. Kalinowski, M. Cocchi, D. Virgili, P. di Marco, V. Fattori, Chem. Phys. Lett. 380 (2003) 710-715.

[4] A. H. Davis, K. Bussmann, J. Vac. Sci. Technol. A 22 (2004) 1885-1891.

[5] T. L. Francis, O. Mermer, G. Veeraraghavan, M. Wohlgenannt, New J. Phys. 6 (2004) 185.

[6] U. Niedermeier, W. Sarfert, H. von Seggern, Proc. of SPIE Vol. 6999 (2008) 699913.

[7] T. D. Nguyen, Y. Sheng, J. E. Rybicki, M. Wohlgenannt, Phys. Rev. B 77 (2008) 235209.

[8] U. Niedermeier, M. Vieth, R. Patzold, W. Sarfert, H. von Seggern, Appl. Phys. Lett. 92 (2008) 193309. 
[9] S. Nowy, W. Ren, A. Elschner, W. Lövenich, W. Brütting, J. Appl. Phys. 107 (2010), 054501.

[10] P. Desai, P. Shakya, T. Kreouzis, W. P. Gillin, N. A. Morley, M. R. J. Gibbs, Phys. Rev. B 75 (2007) 094423.

[11] V. V. Jarikov and D. Y. Kondakov, J. Appl. Phys. 105 (2009) 034905.

[12] D. Y. Kondakov, J. R. Sandifer, C. W. Tang, and R. H. Young, J. Appl.Phys. 93 (2003), 1108.

[13] P.Desai, P. Shakya, T. Kreouzis, and W.P. Gillin, J. Appl. Phys. 102 (2007), 073710.

[14] P. A. Bobbert, T. D. Nguyen, F. W. A van Oost, B. Koopmanns, and M. Wohlgenannt, Phys. Rev. Lett. 99 (2007), 216801.

[15] W. Wagemanns, F. L. Bloom, P. A. Bobbert, M. Wohlgenannt, and B. Koopmanns, J. Appl. Phys. 103 (2008), 07F303.

[16] W. Wagemanns and B. Koopmans, Phys. Status Solidi B (2010), 1-13.

[17] P. Desai, P. Shakya, T. Kreouzis, and W.P. Gillin, Phys. Rev. B 46 (2007), 235202.

[18] S. A. Bagnich, U. Niedermeier, C. Melzer, W. Sarfert, and H. von Seggern, J. Appl. Phys. 106 (2009), 113702.

[19] M. S. Meruvia, J. A. Freire, I. A. Hmmelgen, J. Gruber, and C. F. O. Graeff, Org. Elec. 8 (2007), 695-701. 
[20] T. L. Francis, O. Mermer, G. Veeraraghavan, and M. Wohlgenannt, New J. Phys. 6 (2004), 185.

[21] P. Shakya, P. Desai, M. Somerton, G. Gannaway, T. Kreouzis, and W.P. Gillin, J. Appl. Phys. 103 (2008), 103715.

[22] H. Aziz, Z. D. Popovic, N.-X. Hu, A.-M. Hor, and G. Xu, Science 283 (1999), 1900.

[23] P. A. Bobbert, T. D. Nguyen, W. Wagemans, F. W. A van Oost, B. Koopmanns, and M. Wohlgenannt, Synth. Met. 160 (2010), 223-229. 